THEODORE J. LOWI

The End of Liberalism - The Second Republic of the United States

Second Edition, W. W. Norton and Co, New York, London, 1979.

Der Verf. verficht mit Verve die Auffassung, daß in den USA der atomare, auf Automatismen setzende Liberalismus des 19. Jahrhunderts durch einen kollektivistisch-interventionistischen Liberalismus verdrängt worden ist (welch letzterer sich aber auch seinerseits wieder dem Ende zuneigt; so S. 298: ,, The Second Republic, the regime of interest group liberalism, is finished".) Wie dieses Zitat andeutet, unterscheidet sich die Deutung der Verfassungswirklichkeit durch den Verf. von der landläufigen (,,pressure groups") dadurch, daß für ihn die Gruppen sich den Staat nicht lediglich unterworfen und dienstbar gemacht, sondern sich an seine Stelle gesetzt haben (vgl. vor allem S. 50 ff.). Für das behauptete Ende des individuellen Liberalismus tritt der Verf. zahlreiche gründliche Beweise an, insbesondere durch Schilderung des staatlichen Kampfes gegen die Armut überhaupt (vgl. S. 279: ,,The State of Permanent Receivership"). Für die Verfassung der USA ergibt sich aus dieser Diagnose, daß nicht mehr die geschriebene, sondern die tatsächliche Verfassung des kollektiven Liberalismus in Kraft steht. (S. XI ff.) Als wichtig fallen auf die Ausführungen S. 15 ff.; 19; 37; 78 oben; 91, 105; 138 unten; 185; 215; 232 (Zusammenfassung); 274.

Offenbar will der Verf. provozieren. Aber es handelt sich um eine Provokation, die man sich gern gefallen läßt, weil sie scharfsichtig und durchdringend ist. Insbesondere wer den Bereich des , Offentlichen“ zu erfassen sucht, darf an diesem wesentlichen Buch nicht vorbeigehen.

Herbert Krüger

Hector Fix-Zamudio

Los tribunales constitucionales y los derechos humanos

Instituto de Investigaciones Jurídicas, Serie B, Estudios comparativos, b) Estudios especiales. Número 16, Universidad Nacional Autónoma de México, México 1980, 234 S.

Der Verfasser, der durch seine wegweisenden Veröffentlichungen zur internationalen Menschenrechtsdiskussion über die spanisch sprechende Welt hinaus Beachtung gefunden hat, legt ein Zwischenergebnis seines geplanten umfassenden Werkes über die Verfassungskontrolle in der Welt vor. Eine Kurzbesprechung ist nicht der Ort, um auf seine bemerkenswerten Untersuchungen der Wurzeln der Verfassungsgerichtsbarkeit in den USA und in Osterreich einzugehen. Auch auf die übersichtliche, aber vollständige Darstellung des französischen Systems der Verfassungskontrolle, der Rechtsprechung des deutschen Bundesverfassungsgerichts, der Verfassungsgerichtsbarkeit in Spanien und Portugal, in Jugoslawien und der Tschechoslowakei kann hier nur kurz hingewiesen werden, um einige Worte der Entwicklung der Verfassungsgerichtsbarkeit im engeren Sinne in den Ländern zu widmen, mit denen sich diese Zeitschrift befaßt.

In Lateinamerika haben abweichend von dem amerikanischen System der Verfassungskontrolle durch die ordentlichen Gerichte einige Länder Anstrengungen unternommen, um nach österreichischem Vorbild Gerichte ins Leben zu rufen, die sich auf Verfassungsfragen spezialisieren. In Guatemala wurde das aus Mexiko stammende Amparo-Verfahren 1921 in der Verfassung verankert. 1965 wurde ein besonderer Verfassungsgerichtshof (Corte de la Constitucionalidad) ins Leben gerufen. Dieser tritt zusammen, wenn bestimmte staatliche Stellen oder eine Privatperson, unterstützt von 10 Rechtsanwälten(!), die Verfasssungswidrigkeit eines Gesetzes oder einer Maßnahme der Regierung geltend macht. In Chile wurde 
1970 unter Präsident Frey ein Verfassungsgericht eingeführt, das aber nur von Staatsorganen zur Klärung von Verfassungsfragen, insbesondere der Verfassungsmäßigkeit von Gesetzentwürfen, angerufen werden konnte. Das Verfahren war durch das französische System beeinflußt. Wenn das Gericht auch nur drei Jahre tätig war, entfaltete es umfangreiche Aktivitäten. Nicht weniger als 17 Verfassungsstreitigkeiten zwischen Präsident Allende und dem Kongreß hatte es in eineinhalb Jahren zu entscheiden. In Kolumbien besteht seit 1969 ein Senat des Obersten Gerichtshofes, der auf Verfassungsfragen spezialisiert ist. Auf Grund einer Verfassungspopularklage kann der Oberste Gerichtshof ein Gesetz für ungültig erklären. In Ecuador wurde 1979 ein Tribunal de Garantias Constitucionales geschaffen. Das Gericht hat in Amparo- und Habeas-Corpus-Verfahren zu entscheiden. Es kann Gesetze und Dekrete für vollständig oder teilweise verfassungswidrig erklären. In diesem Fall fordert es das Parlament auf, das verfassungswidrige Gesetz aufzuheben. Kommt der Gesetzgeber dieser Aufforderung binnen 45 Tagen nicht nach, erklärt das Gericht selbst das Gesetz für aufgehoben. In Südkorea wurde 1954 ein Verfassungsgericht geschaffen, das Ähnlichkeiten mit dem deutschen Bundesverfassungsgericht aufwies. 1962 wurde die Verfassungsgerichtsbarkeit wesentlich eingeschränkt. Dem Obersten Gerichtshof wurde lediglich die Befugnis übertragen, im Rahmen eines konkreten Rechtsstreites abschließend über die Verfassungswidrigkeit eines Gesetztes zu befinden. Eine ähnliche Entwicklung ließ sich in Süd-Vietnam bis zum Abzug der amerikanischen Truppen feststellen. Auch hier bestand die Möglichkeit, daß wie in den USA die ordentlichen Gerichte im Verlaufe eines konkreten Rechtstreites ein Gesetz auf seine Verfassungsmäßigkeit zu überprüfen. Ein Oberes Verfassungsgericht sieht auch eine Bestimmung der Verfassung des Irak von 1968 vor, das die Verfassung auszulegen und Verfassungsverletzungen durch die Verwaltung festzustellen hat.

Hans-Rudolf Horn

Carlos M. Oronoz Santana

\section{Manual de Derecho Procesal Penal}

México, D. F., 1978,. 157 S., 82,- mex. Pesos

Dieses Kurzlehrbuch von Oronoz Santana für Jurastudenten stellt in einfachen und einprägsamen Worten das geltende mexikanische Strafprozeßrecht dar, und zwar - was für den nichtmexikanischen Leser nicht so deutlich wird - das für die Bundesinstanzen, insbesondere für den Distrito Federal nach Maßgabe des Código de Procedimientos Penales para el Distrito Federal. Die Gesetzgebungszuständigkeit für das Straf- und Straf prozeßrecht liegt nach dem mexikanischen Verfassungssystem bei den Einzelstaaten ${ }^{1}$. Indessen hat die Bundesverfassung von 1917 in den Art. 14 (Abs. 3: nulla poena sine lege), 16 (Zuständigkeit der Justiz auf verfahrensmäßiger Grundlage) und 19 (Voraussetzungen für Haftdauer von mehr als drei Tagen) mit grundrechtlichen Gewährleistungen die einem Rechtsstaat adäquaten Grenzen gezogen. Art. 20 geht darüber hinaus und legt in zehn Absätzen bis ins einzelne strafprozessuale Bestimmungen fest, etwa auch die, daß eine Gefängnisstrafe nicht wegen Nichtzahlung des Verteidigerhonorars verlängert werden darf. Das Kurzlehrbuch verweist hierauf nur, S. 19/20, 74, der Verfassungstext ist ausführlich genug. Schwerpunkt der Arbeit ist die Darstellung des Beweisrechts einschließlich gewisser Verweisungen (S. 87/8) wie hinsichtlich der öffentlichen Urkunden auf die Legaldefinition in Art. 129 des Código de Procedimientos Civiles para el D. F. (fast wörtlich dem $\$ 415$ Abs. 1 ZPO entsprechend). Interessant für den Leser dieser Zeitschrift ist allerdings wohl nur die Darstellung der Geschichte

1 Vgl. dazu W. Enders, Der mexikanische Bundesstaat, Frankfurt a. M. - Bern - Las Vegas 1977, S. $166 \mathrm{f}$. 